\title{
FAUNA DE DIPTEROS NECRÓFAGOS E SUAS RESPOSTAS À COMPLEXIDADE VEGETAL
}

\author{
MOREIRA, Edimar Agnaldo ${ }^{1}$ edimarbio@ hotmail.com.; PINTO, Gabriel Silva ${ }^{2}$; NEVES, \\ Lilian Cristina Rocha Silva ${ }^{3}$; MARTINS, Cleiton Antônio ${ }^{4}$.
}

1, 2. Docentes do Curso de Ciências Biológicas, Faculdade de Ciências e Tecnologias de Campos Gerais FACICA-MG,

3, 4. Discentes do Curso de Ciências Biológicas, Faculdade de Ciências e Tecnologias de Campos Gerais FACICA-MG

Recebido em: 19/05/2014 - Aprovado em: 30/06/2014 - Disponibilizado em: 30/07/2014

RESUMO. O estudo da dipterofauna necrófaga foi realizado no município de Campo do Meio, Minas Gerais, em 8 áreas distribuídas pela região. A coleta foi realizada com armadilhas específicas para a captura desses insetos, sendo que houve uma busca pelo método que simulasse as condições do interior de uma carcaça (luminosidade e apodrecimento). Nas amostragens, foram priorizados mesmos espaçamentos entre armadilhas para que a escolha do atrativo se encaixasse Teoria do Forrageamento Ótimo. Foram amostrados 286 indivíduos divididos em 3 famílias ou 8 morfoespécies. A matriz de café, teve a maior diversidade encontrada, devido suas características de paisagem. Em termos de atratividade, o fígado em decomposição apresentou maior preferência pela dipterofauna em ambos os pontos de amostragem.

PALAVRAS-CHAVE: Diperofauna, matriz, fragmentação.

ABSTRACT. The study of scavenger dipterofauna was held in Campo do Meio, Minas Gerais, in 8 areas distributed throughout the region. Data collection was performed with specific traps for catching these insects, and there was a search method to simulate conditions inside a (luminosity and rotting) housing. In sampling, the same spacing between traps that the choice of attractive would fit Optimal Foraging Theory were prioritized. 286 individuals divided into 3 families or 8 morphospecies were sampled. The array of coffee, had the highest diversity, because its landscape features. In terms of attractiveness, liver decaying showed greater preference for dipterofauna in both sampling points.

KEYWORDS. Dipterofauna, matrix, fragmentation.

\section{Introdução}

Dentre todos os invertebrados, e mesmo os artrópodes, a classe Insecta é a mais diversa. Estes animais são revestidos por um exoesqueleto quitinoso, apresentando morfologicamente três pares de pernas, um par de antenas e o corpo dividido em cabeça, tórax e abdômen. Apresenta formas aladas e não aladas (TRIPLEHORN; JOHNSON, 2005). Os insetos da Ordem Díptera (moscas e mosquitos) são os principais consumidores de carcaças e isso acarreta 
sua elevada relevância quanto à entomologia forense. As famílias Calliphoridae e Sarcophagidae destacam se pela sua abundância na forma como decompõem os cadáveres (BORNEMISSZA, 1957).

Os dípteros são utilizados normalmente em estudos de entomologia forense com o intuito de determinar o intervalo pós-morte. Essa estimativa é feita a partir do ciclo biológico do inseto que é limitado pelas condições do cadáver (OLIVEIRA-COSTA, 2007). Para a detecção das carcaças utilizadas em sua alimentação, ou como substrato na criação de sua prole, os insetos contam com um aparato sensorial. A atividade destes insetos é regulada por fatores externos como o estágio da decomposição, o período do dia e as condições ambientais (SIMON et al., 1999).

Tanto dípteros quanto outros insetos apresentam especificidades de habitats, com isso as características de um local podem influenciar na população ali presente. Como exemplo, vale ressaltar a formação vegetal que pode interferir na localização de recursos para as populações de insetos, além de proporcionar discrepâncias na umidade e temperatura (RINGEL, 1993), que são fatores cruciais na distribuição de invertebrados (CANELA et al., 2001).
Dentre estes insetos destacam-se os principalmente os caliphorideos que são veiculadores de patógenos (GREENBERG, 1973) e causadores de miíases em vertebrados vivos (GUIMARÃES et al., 1983). Esses insetos apresentam sinantropia, pois possuem grande capacidade de adaptação rápida ao ambiente modificado pelo homem (FURUSAWA; CASSINO, 2006).

No contexto da conservação biológica o tema mais discutido é a fragmentação florestal devido a seu grande efeito sobre a biodiversidade, que são ocasionados principalmente pela redução da área florestal, que resulta em sérias consequências para as comunidades (MYERS et al., 2000). No caso da Mata Atlântica, que é um exemplo significativo de bioma tropical fragmentado, apresenta um dos maiores índices de diversidade biológica e endemismo (MYERS et al., 2000). A criação de áreas abertas nas florestas naturais com finalidade relacionada à agricultura, desenvolvimento urbano, entre outros fins, resulta na criação de paisagens fragmentadas com microclimas distintos causado pela alteração da cobertura vegetal, tais mudanças provocam o declínio da diversidade (RODRIGUES; MARCHINI, 2000, PRIMACK; RODRIGUES, 2001).A relevância deste conhecimento está ligada a maior e melhor compreensão do 
funcionamento da relação diversidade e ecossistema (RONQUI; LOPES, 2006).

Diversos grupos de animais não se arriscam em áreas abertas independente da proximidade dos remanescentes florestais, ficando assim isolados. Com essa situação as populações apresentam uma maior sensibilidade a oscilações em seu número de espécimes, tendo assim maiores riscos à extinção (TURNER, 1996). Tendo como incógnita o efeito de diferentes matrizes agrícolas sobre a diversidade de dípteros necrófagos, objetivou-se com este trabalho estudar a distribuição desses insetos em áreas com distintas complexidades vegetais.

\section{Materiais e Métodos}

O trabalho foi realizado em 8 áreas localizadas na região de Campo do Meio MG (21 $25^{\prime} 45^{\prime}$ ' S e 45\%49'39.95', O), são pontos divididos em 2 tipos de matriz: café e pasto. Para escolha destes, foi considerado o fator da complexidade vegetal do local. A região de Campo do Meio se caracteriza por apresentar os dois tipos de florestas: Estacional Semidecidual e Ombrófila Mista. O clima da cidade é ameno com estações bem definidos, apresentando índice médio pluviométrico anual de 1.592,7 mm. Possui área de 273,8 $\mathrm{Km}^{2}$, com altitude média de $780 \mathrm{~m} \mathrm{e}$ máxima de $1.083 \mathrm{~m}$ na Serra Santa Catarina.
Os dípteros foram coletados com o uso de armadilhas confeccionadas com garrafas pet dois litros, com $20 \mathrm{~cm}$ de altura por $9 \mathrm{~cm}$ de diâmetro, na cor preta, com duas aberturas tipo veneziana, confeccionada no terço inferior, para permitir a entrada dos insetos.

$\mathrm{Na}$ parte superior/interior da armadilha, foi acoplado um funil construído com o bico da garrafa pet com a base voltada para baixo e envolvido em um saco plástico, método semelhante ao utilizado por Ferreira (1978). Dois tipos de iscas foram utilizados: fígado bovino e peixe, apodrecidos.

Para evitar influência da coloração e simular o ambiente de uma carcaça, foram colocadas quatro armadilhas pretas (FERRAZ; AGUIAR-COELHO, 2008) em cada área totalizando 32 armadilhas. Com quatro metros entre si e a aproximadamente 1,8 metros do solo de forma que, em cada área, as armadilhas posicionem-se como se ocupassem os vértices de um quadrado (Figura 1). Foram instaladas no local no período da manhã e a retirada dos indivíduos foi feita em intervalos de 48 horas. 


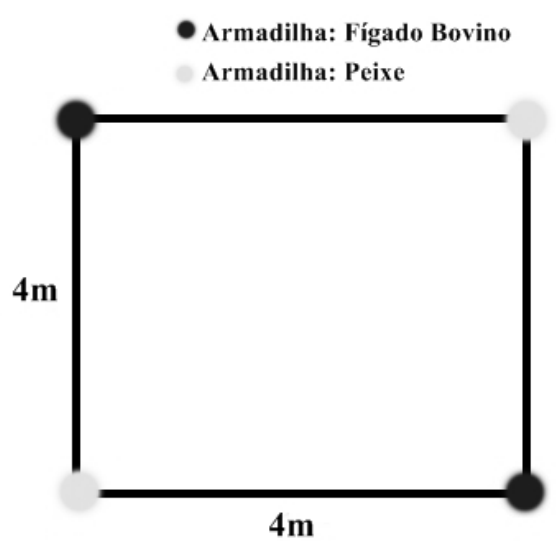

Figura 1. Disposição das armadilhas.

Em seguida, os indivíduos coletados foram classificados a nível de família com as chaves de identificação proposta por Carrera (1967) e Neto et al. (2008) e separados por morfoespécies. A diversidade das áreas pesquisadas foi avaliada por meio do Índice de ShannonWeaver. Para correlacionar a diversidade de dípteros necrófagos com o tipo de matriz a qual foi coletada, foi utilizada a análise de regressão linear múltipla de Dummy ajustando o modelo de regressão em nível de confiança a 95\%. Para análise, as variáveis explicativas (qualitativas Dummy) serão o tipo de matriz e isca. Já a variável resposta foi à diversidade das morfoespécies amostradas. Para os testes de normalidade dos dados e independência dos resíduos foram utilizados os testes Shapiro-Wilk e Box-Pierce, respectivamente. Para a montagem da coleção foram seguidos os padrões estabelecidos por Almeida et al. (1998).

\section{RESULTADOS E DISCUSSÃO}

Durante o trabalho três famílias de dípteros necrófagos foram identificadas: Muscidae, Caliphoridae e Tachinidae, totalizando 286 indivíduos coletados em 8 amostragens, sendo quatro em cada área de estudo. Adotando o critério, separação por coloração e características morfológicas dentro de cada família, foram coletados oito morfoespécies.

A análise de regressão simples foi utilizada para verificar a influência da amostragem na riqueza, constatando que quanto maior o número de amostragens, maior será a riqueza de morfoespécies coletadas. Contudo, ocorreu uma estabilização, não aumentando a riqueza mesmo com aumento das amostragens. A diversidade calculada pelo índice de Shannon-Weaver apontou a monocultura de café como a mais diversa $\left(H^{\prime}=0,3977\right)$ e a matriz de pasto $\left(\mathrm{H}^{\prime}=0,3497\right)$.

A matriz de café apresenta uma cobertura vegetal um pouco mais favorável para a ocupação destes insetos, por apresentar relativa semelhança com um habitat natural, o que não ocorre na matriz de pasto. Isso ocorre devido a fatores que modificam o ambiente no entorno dos fragmentos, ou seja, na matriz sendo este um ambiente inóspito para as populações que ali ocupam (SCARIOT, 2003), pode influenciar na frequência de movimentação 
e ocupação das comunidades na matriz, aumentando as consequências a nível populacional primariamente, pois as condições ambientais são potencialmente desfavoráveis (FAHRIG, 2003).

Ocorre também uma diminuição na diversidade biológica (GURD et al., 2001, STEFFAN-DEWENTER et al., 2002), e redução e distribuição das populações (HANSKI et al., 1996). Dependendo da paisagem em sua totalidade e a qualidade da matriz, esta pode facilitar, dificultar ou até mesmo impedir a movimentação e ocupação. Contudo algumas espécies podem até mesmo utilizar a matriz como habitat alternativo principalmente indivíduos perderam o habitat natural através da fragmentação de hábitats (ESTRADA et al., 1993; MEDELLIN; EQUIHUA, 1998).

Ao verificar a influência do tipo de isca e do local de coleta sobre a diversidade de dípteros necrófagos, foi realizada a análise de correlação de Pearson, sendo que ao avaliar diversidade e tipo de isca a correlação foi de 0,83 com pvalor $(\mathrm{p}<0,001)$, a um nível de significância de 95\%. Quando avaliada a correlação entre diversidade e formação vegetal a correlação foi de 0,51 p-valor $(\mathrm{p}=0,04)$, não sendo uma forte correlação entre estas variáveis, que poderia ser explicado, caso fosse feito um maior esforço amostral. Foi constatado pela análise de regressão Dummy que ambas variáveis (isca e local de coleta) são significativas.

Sendo o modelo de regressão Dummy ajustado, apresentou $\mathrm{R}_{\text {aj }}^{2}=0,9647$ p-valor $(\mathrm{p}<0,001)$, ou seja, 96,47\% da variabilidade é explicada pelo modelo utilizado. As pressuposições de normalidade e independência foram aceitas pelos testes de Shapiro-Wilk e Box-Pierce com $\mathrm{p}$-valores $(\mathrm{p}=0,3368)$ e $(\mathrm{p}=0,5585)$, respectivamente.

A hipótese de que o tipo de isca é uma variável significativa foi aceita, ou seja, a isca de fígado bovina apresentou maior atratividade aos dípteros necrófagos $\mathrm{p}$-valor $(\mathrm{p}<0,001)$. Isso pode ser explicado pela Teoria do Forrageamento Ótimo (TFO) proposta pelos ecólogos Robert MacArthur e Eric Pianka em 1966, para nortear estudos relativos sobre comportamento alimentar. De acordo com esta teoria a quantidade de energia gasta na procura, busca e manipulação do recurso não deve ser maior que os benefícios energéticos destes (MACARTHUR; PIANKA, 1966). Ou seja, a busca pelo recurso ocorrerá sempre pelo alimento mais nutritivo e que gaste a menor quantidade de energia para obtê-lo, assim a isca de fígado, seria o recurso proteico mais abundante que a sardinha. Ao colocar as iscas em distâncias iguais, a fauna de 
díptera foi colocada a selecionar qual o recurso mais viável de acordo com a TFO.

Ao passar do pasto para o café, à diversidade também aumentou $(p<0,001)$, essa variação pode estar ligada ao habitat. A plantação de café é uma barreira contra correntes de ar, o que influencia diretamente o voo de dípteros, já no pasto pela ausência dessas barreiras o vento acaba sendo um forte fator a influenciar a permanência destes insetos. Na matriz de pasto os efeitos físicos são mais evidentes pela menor complexidade vegetal, tendo aumento da exposição das margens dos remanescentes florestais e matriz às ações do vento mais acentuadas, altas temperaturas, queda de umidade e intensificação de radiação solar. Já os efeitos biológicos, ligam-se as mudanças na estrutura das comunidades (MURCIA, 1995).

Outros fatores que influenciam a permanência são: clima, solo e tipo de vegetação. Assim, as alterações causadas pelo desmatamento de mata nativa, podem levar à perda de diversidade alterando a comunidade destes insetos (FAVILA; HALFFTER, 1997). A matriz pode ser definida como a área que recobre a maior extensão da paisagem em questão, apresentando maior conectividade (FORMAN, 1995; METZGER, 2001) podendo ser uma área distinta do fragmento florestal, apresentando diferentes tipos de cobertura e usos (DAVIES et al. 2001; LINDENMAYER; FRANKLIN, 2002), assim essas diferentes coberturas, sendo também chamadas de complexidade vegetal pode influenciar diretamente na ocupação e transição da fauna.

Vários fatores podem estar associados ao deslocamento e ocupação, como os intrínsecos (tamanho corporal, capacidade perceptual, comportamento), sendo que esse fator de percepção está relacionado com a distância que o indivíduo percebe entre pontos do seu deslocamento (ZOLLNER; LIMA, 1999) e também os extrínsecos (características da paisagem, variáveis climáticas e ambientais) que podem favorecer ou não o deslocamento na matriz (PREVEDELLO; VIEIRA, 2010).

Sobre a dominância das famílias amostradas não se pode inferir sobre qual é mais abundante em ambas as áreas, uma vez que foram amostrados apenas indivíduos adultos. Por isso, pelo comportamento reprodutivo de algumas famílias que não necessitam ter contato direto com o recurso para depositar ovos, dificulta sua amostragem (DENNO; COTHRAN, 1975). Para isso, seria necessário um estudo utilizando as iscas da coleta até que emergissem novos adultos e assim poder confirmar a dominância do táxon. 


\section{CONCLUSÕES}

A diversidade da comunidade de dípteros necrófagos foi influenciada pela complexidade vegetal das áreas de estudo, bem como a atratividade foi maior para iscas compostas por fígado bovino. O tipo de vegetação e a qualidade do local em termos de complexidade vegetal são fatores relevantes para movimentação e ocupação de qualquer comunidade biológica, portanto estudos que visem aumentar o conhecimento sobre essa vertente são de grande valia para programas de conservação biológica.

Nesse estudo foram considerados nas amostras apenas indivíduos adultos, para um melhor entendimento da dinâmica das comunidades de díptera, estudos que abordam a identificação dos indivíduos derivados dos ovos presentes nas iscas poderiam corroborar os resultados.

\section{REFERÊNCIAS BIBLIOGRÁFICAS}

BORNEMISSZA, G.F An analysis of arthropod succession in carrion and the effect of its decomposition on the soil fauna. Australian Journal of Zoology, 1957 v. 5 , p. 1-12.

CANELA, M. B. et al. Forrageamento por abelhas Centris spp. (Anthophoridae) em flores de Byrsonima orbignyana A. Juss (Malpighiaceae) no Pantanal Sul. In
Camargo, G. et al. (Eds.) 2001. Ecologia

do Pantanal - Curso de Campo. Editora

Oeste.

DAVIES, K.F.; GASCON, C. \&

MARGULES, C.R. Habitat fragmentation: consequences, management, and future research priorities. In: Soule, M.E. \& Orians, G.H. (Eds.), Conservation Biology: Research Priorities for the Next Decade. Island Press, Washington, pp. 8197. 2001.

DENNO, R.F. \& COTHRAN, W.R. NICHE relationships of a guild of necrophagous flies. Annals of the Entomological Society of America, v.68, p.741-745, 1975.

ESTRADA, A. R.; COATES-ESTRADA, D.; MERITT, JUNIOR., S. \& MONTIEL, D. C. Patterns of frugivore species richness and abundance in forest island and in agricultural habitats at Los Tuxtlas, Mexico. Vegetatio, Dordrecht, v. 107/108, p. 245-257, 1993.

FAHRIG, L. Effects of habitat fragmentation on biodiversity. Annual Reviews of Ecology and Systematics, Palo Alto, v. 34, p. 487-515. 2003.

FAVILA, M. E. \& G. HALFFTER. The use of indicator groups for measuring 
biodiversity as related to community

structure and function. Acta Zoológica

Mexicana. v.72, n.72, p.1-25, 1997.

FERRAZ, A. C. P. \& AGUIAR-COELHO

V. M. Desenvolvimento e Avaliação de

Novas Metodologias para Testar a

Atratividade de Chrysomya megacephala

(Fabricius) (Díptera: Calliphoridae) a

Estímulo Visual por Cores em Condições

Laboratoriais. Neotropical Entomology

37: 2008. 334-337.

FORMAN, R. T. T. Some general

principles of landscape and regional

ecology. Landscape Ecology, 10(3), 133-

142. Gascon, C.; Lovejoy, T.E.;

BIERREGAARD JR., R.O.; MALCOLM, J.R.; STOUFFER, P.C.; VASCONCELOS, H.L.; LAURANCE, W.F.; ZIMMERMAN, B.; TOCHER, M. \& BORGES, S. (1999).

Matrix habitat and species richness in tropical forest remnants. Biological

Conservation 91: 223-229. 1995.

FURUSAWA, G. P. \& CASSINO P. C. R.

Ocorrência e distribuição de Calliphoridae

(Díptera: Oestroidea) em um Fragmento de

Mata Secundária no Município de Paulo de Frontin, Médio Paraíba, RJ. Revista de

Biologia e Ciências da Terra 6: 2006. 152-164.
GREENBERG, B. Flies and disease:

biology and disease transmission. New

Jersey, Princeton University, 1973. 447 p.

GUIMARÃES J. H.; PAPAVERO N. A. \&

PRADO A. P. As miíases na região

Neotropical (identificação, biologia e

bibliografia). Revista Brasileira de

Zoologia 1: 1983. 293-416.

GURD, D.B.; NUDDS, T.D. \& RIVARD,

D.H. Conservation of mammals in eastern

North American wildlife reserves: how

small is too small. Conservation Biology,

15: 1355-1363. 2001.

HANSKI, I.; MOILANEN, A. \&

GYLLENBERG, M. Minimum viable

metapopulation size. American

Naturalist, 147: 527-41. 1996.

LINDENMAYER, D.B. \& FRANKLIN,

J.F. Conserving Forest Biodiversity. A

Comprehensive Multiscale Approach.

Island Press, Washington. 2002.

MACARTHUR, R.H. \& PIANKA, E.R.

On optimal use of a patchy environment.

American Naturalist, 100: 603-609. 1966.

MEDELLIN, R. A. \& EQUIHUA, M.

Mammal species richness and habitat use

in rainforest and abandoned agricultural

fields in Chiapas, Mexico. Journal of 
Applied Ecology, Oxford, v. 35, p. 13-23, 1998.

METZGER, J.P. O que é ecologia de paisagens. Biota Neotropica, 1: 1-9. 2001.

MURCIA, C. Edge effects in fragmented forests: implications for conservation.

Trends in Ecology and Evolution. 10:58-

62. 1995.

MYERS, N.; MITTERMEIER, R. A.; MITTERMEIER, C. G.; FONSECA, G. A. B. \& KENT, J. Biodiversity hotspots for conservation priorities. Nature, London, v. 403, n. 6772, p. 853 - 858, fev. 2000.

\section{OLIVEIRA-COSTA, J. Entomologia}

Forense - Quando os insetos são vestígios. Campinas: Millennium, 2007, p. 29-49

PREVEDELLO, J.A. \& VIEIRA, M.V.

Does the type of matrix matter: A quantitative review of the evidence.

Biodiversity and Conservation, 19:12051223. 2010.

PRIMACK, R. B. \& RODRIGUES, E. Biologia da Conservação. Londrina, Ed. Planta, 328 p. 2001.

RINGEL, M. S. The effects of forest fragmentation on communities of ground-foraging arthropods in central Amazonia. Monografia, Bacharelado em Artes, Universidade de Princeton 1993.

RODRIGUES, S. R. \& MARCHINI, L. C.. Ocorrência de besouros coprófagos em dois diferentes ambientes. Revista Brasileira de Entomologia 44: 35-38. 2000.

RONQUI, D.C. \& LOPES, J. Composição e diversidade de Scarabaeoidea (Coleoptera) atraídos por armadilha de luz em área rural no norte do Paraná, Brasil.

Iheringia Ser. Zool. 96 (1):103-108. 2006.

SIMON, M. A fauna associada a carcaças na Amazônia Central: caracterização e sucessão degradativa. pp. 78-81 In: Curso de Campo Ecologia da Floresta Amazônica, Venticinque, E. \& Hopkins, M. PDBFF, Manaus 1999.

STEFFAN-DEWENTER, I.;

MÜNZENBERG, U.; BÜRGER, C.; THIES, C. \& TSCHARNTKE, T. Scaledependent effects of landscape context on three pollinator guilds. Ecology, 83: 14211432. 2002.

TRIPLEHORN, C. A. \& JOHNSON. N. F. BORROR AND DELONG'S

Introduction to the Study of Insects. 7th 
edition. Thomson Brooks / Cole, Belmont, 2005864 p.

TURNER, I. M. Species loss in fragments

of tropical rain forest: a review of the evidence. Journal of Applied Ecology, Oxford, v. 33, n. 2, p. 200-209. 1996.

ZOLLNER, P.A. \& LIMA, L.S. Search strategies for landscape-level interpatch movements. Ecology, 80: 1019-1030. 1999. 